\title{
A CRITICAL-POINT YIELD MODEL TO APPRAISE THE DAMAGE CAUSED TO SOYBEAN BY WHITE-MOLD
}

\author{
MODELO DE PONTO CRÍTICO PARA ESTIMAR OS DANOS CAUSADOS PELO \\ MOFO-BRANCO EM SOJA
}

\section{Erlei M. REIS ${ }^{1}$; Mateus ZANATTA ${ }^{1}$; Fernando Cezar JULIATTI ${ }^{\mathbf{2}}$; Hercules D. CAMPOS ${ }^{\mathbf{3}}$; Luis Henrique Carregal P. SILVA ${ }^{3}$; Maurício C. MEYER ${ }^{4}$; José NUNES JUNIOR ${ }^{4}$; Cláudia B. PIMENTA ${ }^{5}$; Daniel CASSETARI NETO ${ }^{5}$; Andréia Q. MACHADO ${ }^{6}$; Carlos M. UTIAMADA ${ }^{7}$}

1. Instituto AGRIS, Paso Fundo, RS, Brasil. erleireis@tpo.com.br; 2. Universidade Federal de Uberlândia - UFU, Instituto de Ciências Agrárias - ICIAG, Uberlândia, MG, Brasil. juliatti@ufu.br; 3. Universidade de Rio Verde, Rio Verde, GO; 4. Embrapa Soja/CTPA/Emater - Goiânia, GO, Brasil; 5. Universidade Federal de Mato Groso/UNIVAG - Cuiabá, MT, Brasil; 6. Tagro - Londrina, PR, Brasil.

\begin{abstract}
A model to estimate the damage caused by white mold to soybean yield from experimental field data gathered during the summer season of 2009-10 was generated. Six soybean cultivars were grown on six sites of the Savana (Cerrados) region, resulting in a total of nine separate experiments. The gradient of disease intensity (plant stem incidence) and yield was generated through the application of different fungicides and rates three times over the course of the season. The disease incidence in plant stems was evaluated at the R1, R5.2 and R5.5 growing stages. Manual harvest at the physiological ripening stage was followed by grain drying, threshing, and cleaning. Finally, grain yield was estimated in $\mathrm{kg} / \mathrm{ha}$, and regression analysis was performed. Nine linear equations representing the damage function were generated. The mean damage function was $\mathrm{y}=-6.7 \mathrm{x}+1,000$, where y represents grain yield normalized to $1,000 \mathrm{~kg} / \mathrm{ha}$ and $\mathrm{x}$ represents WM incidence in plants. To appraise the damage caused by various disease intensities, these models should first be validated. Damage coefficients may be used to determine the level of economic damage.
\end{abstract}

KEYWORDS: Glycine max. Sclerotinia sclerotiorum. Sclerotinia stem rot.

\section{INTRODUCTION}

The Brazilian soybean [Glycine $\max (\mathrm{L}$.) Merrill] crop cultivated in 2018/19 covered an area of 35 million hectares and yielded $2.8 \mathrm{t} / \mathrm{ha}$, resulting in an overall production of 50 million tons (CONAB, 2019). The disease complex in soybean causes reductions in crop yield. The main soybean stem diseases are anthracnose (Colletotrichum truncatum Andrus \& Moore), meridionalis-canker (Diaporthe aspalathi Janse van Rensburg, Castlebury \& Crous.), caulivora-canker [Diaporthe caulivora (Athow \& Caldwell) Santos], pod and stem blight [Phomopsis phaseoli (Desmaz.) Sacc.], brown pith rot [Cadophora gregata (Allington \& Chamberlain) Arlington \& McNew], and white mold (WM) caused by Sclerotinia sclerotiorum (Lib.) de Bary (ABAWI; GROGAN, 1979; FARIAS NETTO et al., 2008; HARTMAN; SINCLAIR; RUPE, 1999;). Sclerotinia stem rot is one of the most devastating soybean diseases in the central states of Brazil, where up to a $20 \%$ reduction in soybean yields due to WM has been reported (FARIAS NETO et al., 2008). In the present work, damage is used according to Nutter, Teng and Royer (1993).

In plant disease epidemics, the damage quantification should be a clear priority; however, only a few reliable studies have quantified the effects of disease on soybean grain yield (BERGAMIN FILHO; AMORIM, 1996).

A number of models have been used to estimate the damage caused by plant diseases. The critical-point model is particularly useful when one can identify a specific stage of plant growth at which disease intensity is highly correlated with future damage. In practice, a simple model can be used to estimate the future damage caused to the host by a specific disease, calculated as a function of the host's phenological stage and the disease intensity (BERGAMIN FILHO; AMORIM, 1996).

In disease damage quantification, it is necessary to generate disease and yield gradients to relate them to each other. Various methods can be used to appraise damage caused by plant disease. For example, the damage caused by wheat scab (REIS et al., 1996), wheat blast (GOULART; PAIVA; MESQUITA, 1992), and corn stem rot (DENTI; REIS, 2003) has been determined without 
the use of fungicide. The disease infection intensity gradient used to estimate damage may also be calculated by varying the fungicide applied, the rates of application, or the number of applications involved (SAH; MCKENZIE, 1987).

In our study, we sought to quantify the deleterious effects of WM infection on soybean yields by generating equations that, when implemented in a critical-point yield model, would predict the future damage as a function of different disease intensities for soybean cultivars. The damage coefficient generated by the model could also be used to determine the economic damage threshold (MUNFORD; NORTON, 1984).

\section{MATERIAL AND METHODS}

The experiments were conducted at six sites using six susceptible soybean cultivars, resulted in a total of nine experiments (Table 1). The six sites were located in altitudes between 891.0 and 1,127.0 $\mathrm{m}$ above sea level (Table 1). All soybean varieties cultivated in Brazil are considered susceptible to WM, although data concerning their susceptibility to the disease is not available (INDICAÇÕES, 2008)

Table 1. Details about location, height, soybean cultivars and seeding date for the 2009-2010 growing season.

\begin{tabular}{|c|c|c|c|c|}
\hline County/state & $\begin{array}{l}\text { Geographic } \\
\text { position }\end{array}$ & $\begin{array}{c}\text { Altitude } \\
\text { (m above sea level) }\end{array}$ & Cultivar & Sowing date \\
\hline Montividiu, GO & $17^{\circ} 25^{\prime} 16^{\prime \prime S}$ & 921 & P98Y11 & $10 / 19 / 2009$ \\
\hline S.M. Passa Quatro, GO & $\begin{array}{l}16^{\circ} 51^{\prime} 46^{\prime \prime ~ S} \\
48^{\circ} 45^{\prime} 12^{\prime \prime} \mathrm{W}\end{array}$ & 1,027 & MSoy 7908 RR & $11 / 03 / 2009$ \\
\hline Água Fria, GO & $\begin{array}{l}14^{\circ} 57^{\prime} 54^{\prime \prime} \mathrm{S} \\
47^{\circ} 46^{\prime} 08^{\prime \prime} \mathrm{W}\end{array}$ & 891 & MSoy 7908 RR & $11 / 09 / 2009$ \\
\hline Campo Verde, MT & $\begin{array}{l}15^{\circ} 06^{\prime} 55^{\prime \prime} \mathrm{S} \\
54^{\circ} 56^{\prime} 17^{\prime \prime} \mathrm{W}\end{array}$ & 985 & MSoy 8230 RR & $10 / 13 / 2009$ \\
\hline Uberlândia, MG & $\begin{array}{l}14^{\circ} 12^{\prime} 54^{\prime \prime} \mathrm{S} \\
47^{\circ} 56^{\prime} 58^{\prime \prime} \mathrm{W}\end{array}$ & 947 & BRS Valiosa RR & $11 / 19 / 2009$ \\
\hline Mauá da Serra, PR & $\begin{array}{l}23^{\circ} 54^{\prime} 26^{\prime \prime} \mathrm{S} \\
51^{\circ} 11^{\prime} 29^{\prime \prime} \mathrm{W}\end{array}$ & 1020 & BRS 232 & $11 / 14 / 2009$ \\
\hline
\end{tabular}

Individual experimental units were composed of four rows $0.5 \mathrm{~m}$ apart, each $6.0 \mathrm{~m}$ long, with the two outside rows as borders. Fungicide was applied with $\mathrm{CO}_{2}$-pressurized knapsack atomizer, which had a boom $2.0 \mathrm{~m}$ long and delivering $200-300 \mathrm{~L} / \mathrm{ha}$. The fungicides were applied four times, in blocks arrangement in a randomized blocks pattern.

At different soybean growth stages (R1, R5.2 and R5.5) (RITCHIE; HANWAY; THOMPSON, 1982), the two central rows in each plot were used to determine the incidence of WM on soybean stems. At physiologic maturity, the two central rows plants were manually harvested, and the grains threshed, dried, cleaned, and weighed, and yield calculated in $\mathrm{kg} / \mathrm{ha}$.

Regression analyses were performed on the grain yield recorded as dependent variable and WM incidence as independent variable for sites. Equations were expressed on the basis of grain yield (y) normalized to $1,000 \mathrm{~kg} / \mathrm{ha}$ in the form $\mathrm{y}=1,000$ $-a(\mathrm{x})$, where $a$ represents the damage coefficient $[\mathrm{kg} / \mathrm{ha} / 1 \%$ plant incidence (hereafter, units not indicated)]. Equations represent the critical-point model sought in the work.

\section{RESULTS AND DISCUSSION}

Fungicides treatments resulted in disease and grain yield gradients (Tables 2 and 3), showing that they may be used in research to determine the damage function for a specific disease (SAH; MCKENZIE, 1987). In the present study, the incidence of disease in plants was determined at three growth stages. WM was not detected at R1 (blooming beginning), confirming that WM only occurs after flowering, as the pathogen requires flowers or senesced petals as infection sites (1). The lowest incidence $(31.6 \%)$ of WM was measured in Água Fria at R5, while the highest incidence (90.3\%) was recorded in Montevidiu at R5.5. 
Table 2. Fungicide treatments used to generate disease and yield gradients.

\begin{tabular}{|c|c|c|c|c|c|c|c|}
\hline \multirow[t]{2}{*}{ Treatments } & \multirow[t]{2}{*}{ Technical name } & \multicolumn{4}{|l|}{ Timing } & \multicolumn{2}{|c|}{$\begin{array}{l}\text { Rate } \\
\mathrm{L} \text { or } \mathrm{kg} / \mathrm{ha}\end{array}$} \\
\hline & & $1^{\mathrm{a}}$ & $2^{\mathrm{a}}$ & $3^{\mathrm{a}}$ & $4^{\mathrm{a}}$ & C.F. & A.I. \\
\hline 1 & Unsprayed & & & & & & \\
\hline \multirow[t]{2}{*}{2} & Methyl tiophanate & - & 10 DAA & 10 DAA & - & 1.0 & 0.5 \\
\hline & Fluazinam & R1 & - & - & - & 1.0 & 0.5 \\
\hline \multirow[t]{2}{*}{3} & Methyl tiophanate & - & 10 DAA & - & - & 1.0 & 0.5 \\
\hline & Fluazinam & R1 & - & 10 DAA & - & 1.0 & 0.5 \\
\hline 4 & Fluazinam & R1 & 10 DAA & & - & 1.0 & 0.5 \\
\hline 5 & Fluazinam & R1 & 10 DAA & 10 DAA & - & 1.0 & 0.5 \\
\hline \multirow[t]{3}{*}{6} & Methyl tiophanate & - & 10 DAA & - & - & 1.0 & 0.5 \\
\hline & Procimidone & R1 & - & - & - & 1.0 & 0.5 \\
\hline & Fluazinam & - & - & 10 DAA & - & 1.0 & 0.5 \\
\hline \multirow[t]{2}{*}{7} & Carbendazim & $10 \mathrm{DBB}$ & - & 10 DAA & - & 1.0 & 0.5 \\
\hline & Fluazinam & - & R1 & - & 10 DAA & 1.0 & 0.5 \\
\hline \multirow[t]{2}{*}{8} & Methyl tiophanate & $10 \mathrm{DBB}$ & - & 10 DAA & - & 1.0 & 0.5 \\
\hline & Fluazinam & - & R1 & - & 10 DAA & 1.0 & 0.5 \\
\hline \multirow[t]{2}{*}{9} & Carbendazim & R1 & - & 10 DAA & - & 1.5 & 0.75 \\
\hline & Fluazinam & - & 10 DAA & - & - & 1.0 & 0.5 \\
\hline
\end{tabular}

$\mathrm{CF}=$ commercial formulation; A.I. = active ingredient; $\mathrm{DBB}=$ days before blooming; $\mathrm{DAA}=$ days after last application

Table 3. Fungicide treatments and application time to generate disease and yield gradients

\begin{tabular}{|c|c|c|c|c|c|c|c|}
\hline \multirow[t]{2}{*}{ Treatments } & \multirow[t]{2}{*}{$\begin{array}{l}\text { Technical } \\
\text { name }\end{array}$} & \multicolumn{4}{|c|}{ Timing } & \multicolumn{2}{|c|}{$\begin{array}{l}\text { Rate } \\
\mathrm{L} \text { or kg/ha }\end{array}$} \\
\hline & & $1^{\mathrm{a}}$ & $2^{\mathrm{a}}$ & $3^{\mathrm{a}}$ & $4^{\mathrm{a}}$ & C.F. & I.A. \\
\hline 1 & Unsprayed & & & & & & \\
\hline 2 & Methyl tiophanate & R1 & 10 DAA & 10 DAA & 10 DAA & 1.0 & 0.5 \\
\hline 3 & Carbendazim & R1 & 10 DAA & 10 DAA & 10 DAA & 1.0 & 0.5 \\
\hline 4 & Procimidone & R1 & 10 DAA & - & & 1.0 & 0.5 \\
\hline 5 & Fuazinam & R1 & 10 DAA & - & & 1.0 & 0.5 \\
\hline 6 & Fluazinam & R1 & 10 DAA & 10 DAA & & 1.0 & 0.5 \\
\hline 7 & Fluopyram & R1 & 10 DAA & - & & $0.4+0.4$ & 0.2 \\
\hline 8 & Fluopyram & R1 & 10 DAA & 10 DAA & & $0.4+0.4$ & 0.2 \\
\hline 9 & Dimoxystrobin+boscalid & R1 & 10 DAA & - & & 1.0 & 0.4 \\
\hline 10 & Dimoxystrobin+boscalid & R1 & 10 DAA & 10 DAA & & 1.0 & 0.4 \\
\hline 11 & Penthiopyrad & R1 & 10 DAA & & & 2.5 & 0.5 \\
\hline 12 & Penthiopyrad & R1 & 10 DAA & 10 DAA & & 2.5 & 0.5 \\
\hline * Added Ni & us $500 \mathrm{~mL} / \mathrm{ha}$. & & & & & & \\
\hline
\end{tabular}

The relationship between WM incidence and soybean yield was described well by linear regression models, with coefficients of determination $\left(\mathrm{R}^{2}\right)$ ranging from 0.56 to 0.88 (Figs. 1 and 2). In the experiments, the lowest incidence was $31.6 \%$, the highest was $90.3 \%$, and the overall mean was $45.5 \%$. Damage coefficients varied from 3.9 to $13.0 \mathrm{~kg} / \mathrm{ha}$ for a $1,000 \mathrm{~kg} / \mathrm{ha}$ grain yield, and the overall damage function was described by the function $\mathrm{y}=-6.7 \mathrm{x}+1,000$, meaning that there was a grain yield reduction of $6.7 \mathrm{~kg} / \mathrm{ha}$ to $1,000 \mathrm{~kg} / \mathrm{ha}$ yield for each 1\% WM plant incidence (Table 4). The average damage calculated in the experiments (Table 4) was $273.2 \mathrm{~kg} / \mathrm{ha}$ ranging from $120.8 \mathrm{~kg} / \mathrm{ha}$ (Água Fria, GO to 403.0 kg/ha Uberlândia, MG). 
A critical-point...

REIS et al.

Table 4. Critical-point equations for grain yield (normalized to $1,000 \mathrm{~kg} / \mathrm{ha}$ ) and damage calculated for each location.

\begin{tabular}{|c|c|c|c|c|c|}
\hline County/state & $\begin{array}{l}\text { Equation } \\
(y=-a x+b)\end{array}$ & $\mathrm{R}^{2}$ & $p$ & $\begin{array}{l}\text { Location } \\
\text { highest } \\
\text { incidence } \\
(\mathrm{I}=\mathrm{x}=\%)\end{array}$ & $\begin{array}{l}\text { Damage }^{y} \\
(\mathrm{~kg} / \mathrm{ha}) \\
\left(\mathrm{a}^{*} \mathrm{x}\right)\end{array}$ \\
\hline $\begin{array}{l}\text { São Miguel do Passa } \\
\text { Quatro }^{\text {(v) }}, \text { GO }\end{array}$ & $\underset{(\mathrm{x})}{\mathrm{Y}^{(\mathrm{z})}}=-5.7 \mathrm{x}+1,000$ & 0.82 & 0.00007 & 36.9 & $210.7^{(\mathrm{y})}$ \\
\hline Montividiu $^{(\mathrm{v})}, \mathrm{GO}$ & $y=-3.9 x+1,000$ & 0.76 & 0.002 & 74.7 & 291.3 \\
\hline Água Fria ${ }^{(v)}, G O$ & $y=-3.8 x+1,000$ & 0.61 & 0.01 & 31.6 & 120.8 \\
\hline Campo Verde ${ }^{(\mathrm{v})}, \mathrm{MT}$ & $y=-6.5 x+1,000$ & 0.56 & 0.02 & 40.6 & 263.9 \\
\hline Uberlândia $^{(\mathrm{v})}, \mathrm{MG}$ & $y=-13.0 x+1,000$ & 0.88 & 0.0002 & 31.0 & 403.0 \\
\hline Mauá da Serra ${ }^{(v)}, \mathrm{PR}$ & $y=-9.0 x+1,000$ & 0.74 & 0.003 & 33.4 & 300.6 \\
\hline Uberlândia $^{(\mathrm{x})}, \mathrm{GO}$ & $y=-9.0 x+1,000$ & 0.69 & 0.0008 & 32.0 & 288.0 \\
\hline $\begin{array}{l}\text { São Miguel do Passa } \\
\text { Quatro }{ }^{(\mathrm{x})}, \mathrm{GO}\end{array}$ & $y=-5.8 x+1,000$ & 0.60 & 0.03 & 39.4 & 228.5 \\
\hline Montividiu ${ }^{\mathrm{x}}$, GO & $y=-3.9 x+1,000$ & 0.72 & 0.0004 & 90.3 & 352.2 \\
\hline Mean & $y=-6.7 x+1,000$ & 0.70 & 0.007 & 45.5 & 273.2 \\
\hline
\end{tabular}

$\left({ }^{v}\right)$ Data generated with respect to treatments outlined in Table $2 ;\left(^{\mathrm{x}}\right)$ data generated with respect to treatments outlined in Table 3; $\left(^{y}\right)$ damage $=a^{*} \mathrm{x}(\mathrm{a}=5.7 * 36.9=210.7 \mathrm{~kg} / \mathrm{ha}) ;\left(^{\mathrm{z}}\right) \mathrm{y}=$ grain yield and $\mathrm{x}=$ white mold plant stem incidence $(\mathrm{I}) ; \mathrm{a}=$ damage coefficient; $\mathrm{b}=$ grain yield normalized to $1,000 \mathrm{~kg} / \mathrm{ha}$.

In Iowa state (USA), YANG, Lundeen and Uphoff (1999) found the relationship between grain yield and WM plant incidence. The damage at $70 \%$ stem incidence was estimated to be $59 \%$, using the linear function $\mathrm{y}=-33.5 \mathrm{x}+3,970$. When yields were normalized to $1,000 \mathrm{~kg} / \mathrm{ha}$, the damage coefficient obtained was $8.43 \mathrm{~kg} /$ ha for each $1 \%$ WM plant incidence. These findings are similar to our general mean of $6.7 \mathrm{~kg} / \mathrm{ha}$ for $1 \%$ plant incidence (Table 4).

\section{CONCLUSION}

WM has the potential to cause great damage to soybean crops. Plant-stem incidence is more reliable than the subjective criterion of severity in estimating damage. The equations relating stem incidence to grain yield can be used to appraise the damage caused by WM in cultivars whose susceptibility was similar to that of those cultivars tested in the present work.

\section{ACKNOWLEDGEMENTS}

Thanks to FAPEMIG for supporting the White Mold Management Project in Minas Gerais and Brazil

RESUMO: Desenvolveu-se um modelo para estimar os danos causados pelo mofo-branco (MB) (Sclerotinia sclerotiorum) na cultura da soja, com dados gerados em experimentos de campo conduzidos na safra de soja de 2009/10. Seis cultivares de soja foram cultivados em seis locais perfazendo um total de nove experimentos em distintas regiões edafoclimáticas na região do Cerrado. O gradiente da intensidade da doença, avaliada em função de incidência de sintomas/sinais em hastes, foi gerado pela aplicação de diferentes fungicidas em momentos e doses distintas. A intensidade da doença foi avaliada, nos estádios fenológicos de R1, R5.2 e R5.5. A colheita foi realizada na maturação fisiológica e o rendimento de grãos expresso em $\mathrm{kg} / \mathrm{ha}$. As análises de regressão entre o rendimento de grãos e a incidência da doença foram realizadas para todas as combinações obtidas e geraram nove equações lineares da função de dano. Função de dano média de nove experimentos foi $\mathrm{R}=1.000$ - 6,7 I (onde $\mathrm{R}=$ rendimento de grãos normalizado para $1.000 \mathrm{~kg} / \mathrm{ha}$ e I incidênciaa do MB em plantas). Para estimar o dano causado por intensidades diferentes da doença, esses modelos devem ser previamente validado. Os coeficientes de dano podem ser usados para determinar o limiar de dano econômico. sclerotiorum.

PALAVRAS-CHAVE: Esclerociniose. Glycine max. Podridão de esclerocímia. Sclerotinia 


\section{REFFERENCES}

ABAWI, G.S.; GROGAN, R.G. Epidemiology of diseases caused by Sclerotinia sclerotiorum. Phytopathology, St. Paul, v. 69, n. 8, p. 899-904, 1979. https://doi.org/10.1094/Phyto-69-899

BERGAMIN FILHO, A.; AMORIM, L. Doenças de plantas tropicais: epidemiologia e controle econômico. São Paulo: Ed. Agronômica Ceres, 1996. 289 p.

CONAB, levantamento de safras. Disponível em: https://www.conab.gov.br/info-agro/safras/graos, boletins grãos setembro 2019. Acesso em setembro/2019.

DENTI, E.A.; REIS, E.M. Levantamento de fungos associados às podridões do colmo e quantificação de danos em lavouras das regiões do Planalto Médio Gaúcho e dos Campos Gerais do Paraná. Fitopatologia Brasileira, Brasília, v. 28, n, 6, p. 585-590, 2003. https://doi.org/10.1590/S0100-41582003000600001

FARIAS NETO, A.L.; DIANESE, A.C.; SOUZA, P.I.M.; MOREIRA, C.T.; ALMEIDA, A.M.R.; SEIXAS, C.D.S. Podridão vermelha-da-raiz e mofo-branco na cultura da soja. Planaltina, DF: Embrapa Cerrados. 2008. 27p. (Doc. 235/Embrapa Cerrados).

GOULART, A.C.P.; PAIVA, F.A.; MESQUITA, N.A. Perdas em trigo causadas por Pyricularia oryzae. Fitopatologia Brasileira, Brasília, v. n. 1, p.115-117, 1992.

HARTMAN. G.L.; SINCLAIR, J.B.; RUPE, J.C. Compendium of soybean disease. Fourth Ed. APS Press, St. Paul, 1999, 100p.

INDICAÇÕES TÉCNICAS PARA A CULTURA DA SOJA NO RIO GRANDE DO SUL E SANTA CATARINA 2008-2009/36 Reunião de Pesquisa da soja da Região Sul, Porto Alegre, RS, 29 a 31 de julho de 2008. Porto Alegre: FEPAGRO, 2008, 144p.

MUNFORD, J. D.; NORTON, G. A. Economics of decision making in pest management. Annual Review of Entomology, Palo Alto, v. 29, p.157-174, 1984. https://doi.org/10.1146/annurev.en.29.010184.001105

NUTTER, F.W.; TENG, P.S.; ROYER, M.H. Terms and concepts for yield, crop loss, and disease thresholds. Plant Disease, St. Paul, v. 77, n. 2, p. 211-215, 1993.

REIS, E.M.; BLUM, M.M.C.; CASA, R.T.; MEDEIROS, C.A. Grain losses caused by infection of wheat heads by Gibberella zeae in southern Brazil, from 1984 to 1994. Summa Phytopathologica, Jaboticabal, v. 22, n. 02, p. 134-137, 1996.

RITCHIE, S; HANWAY, J.J.; THOMPSON, H.E. How a soybean plant develops. Ames, Iowa State University of Science and Technology, Coop. Ext. Serv. 1982. 20p. (Special Report, 53).

SAH, D.N.; MCKENZIE, D.R. Methods of generating different levels of disease epidemics in loss experiments. In: Teng, P.S. (ed.) Crop loss assessment and pest management. St. Paul, MN: The American Phytopathological Society, p.90-95. 1987.

YANG, X.B.; LUNDEEN, P.; UPHOFF, M.D. Soybean varietal response and yield loss caused by Sclerotinia sclerotiorum. Plant Disease, St. Paul, v. 83, p. 456-461, 1999. https://doi.org/10.1094/PDIS.1999.83.5.456 